\title{
Multidisciplinary Taiwan Consensus Recommendations for the Use of DEBDOX-TACE in Hepatocellular Carcinoma Treatment
}

\author{
Pi-Yi Chang ${ }^{a}$ Chun-Chieh Huang $^{b}$ Chao-Hung Hung ${ }^{c}$ Chih-Yung Yu ${ }^{d}$ \\ Ding-Kwo Wu ${ }^{e}$ Jen-I Hwang ${ }^{f}$ Po-Chin Liang ${ }^{2}$ Reng-Hong $\mathrm{Wu}^{\mathrm{h}}$

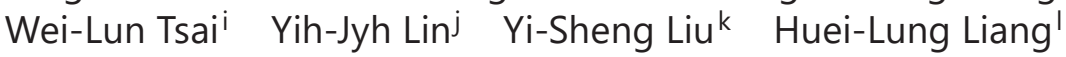 \\ Rheun-Chuan Lee ${ }^{m} \quad$ Chien-Hung Chen ${ }^{n}$ \\ aDepartment of Radiology, Taichung Veterans General Hospital, Taichung City, Taiwan; \\ ${ }^{b}$ Department of Radiology, Far Eastern Memorial Hospital, Taipei, Taiwan; ' ${ }^{\text {Department }}$ \\ of Internal Medicine, Kaohsiung Chang Gung Memorial Hospital, Kaohsiung City, Taiwan; \\ ${ }^{\mathrm{d}}$ Department of Radiology, Tri-Service General Hospital, Taipei, Taiwan; ${ }^{\mathrm{e}}$ Department of \\ Radiology, Kaohsiung Medical University Chung-Ho Memorial Hospital, Kaohsiung City,

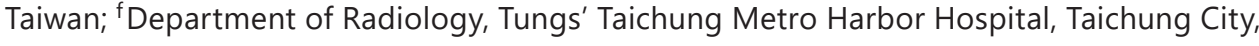 \\ Taiwan; ${ }^{9}$ Department of Radiology, National Taiwan University Hospital, Taipei, Taiwan; \\ hDepartment of Radiology, Chi Mei Hospital, Tainan City, Taiwan; 'Department of Internal \\ Medicine, Kaohsiung Veterans General Hospital, Kaohsiung City, Taiwan; ${ }^{j}$ Department \\ of Surgery, National Cheng Kung University Hospital, Tainan City, Taiwan; ${ }^{k}$ Department \\ of Diagnostic Radiology, National Cheng Kung University Hospital, Tainan City, Taiwan; \\ 'Department of Radiology, Kaohsiung Veterans General Hospital, Kaohsiung City, Taiwan; \\ m Department of Radiology, Taipei Veterans General Hospital, Taipei, Taiwan; ${ }^{n}$ Department \\ of Internal Medicine, National Taiwan University Hospital Yunlin Branch and National Taiwan \\ University College of Medicine, Douliu City, Taiwan
}

\section{Keywords}

Chemoembolization - DEBDOX - Doxorubicin · Drug-eluting bead · Hepatocellular carcinoma

\begin{abstract}
Transarterial chemoembolization (TACE) is the first-line treatment in patients with unresectable hepatocellular carcinoma (HCC). In recent years, there has been increasing clinical evidence that drug-eluting beads provide a combined ischemic and cytotoxic effect that may be superior to conventional TACE, with low systemic toxicity. The therapeutic value of TACE performed using the embolic microsphere DC Bead loaded with doxorubicin (drug-eluting bead
\end{abstract}

All authors contributed equally to this work. The findings of this manuscript have been presented at a symposium in the 2017, 8th Asia Pacific Primary Liver Cancer (APPLE) Meeting, Singapore. 


\section{Liver Cancer}

doxorubicin [DEBDOX]) has been shown by several randomized controlled trials. Since Lencioni et al. [Cardiovasc Intervent Radiol 2012;35:980-985] published the first widely accepted technical recommendations on HCC embolization with DEBDOX-TACE in 2012, new studies have contributed to a better understanding of when and how to apply this new therapeutic modality, and they have yet to be incorporated into an updated guideline. Additionally, differences in the underlying liver pathology and practice of transcatheter embolization between Asian and Western populations have not been adequately addressed, and there remain significant variations in the TACE protocols adopted in different parts of the world. These mainly revolve around the number and type of chemotherapeutic agents used, type of embolic material, reliance on Lipiodol, and selectivity of catheter positioning. As a result of these issues, it has been difficult to interpret and compare results obtained from different centers in a systematic fashion. To address these concerns, we convened a panel of experts specializing in different aspects of HCC treatment to craft an updated set of recommendations that better reflect recent clinical experiences and are tailored to the use of DEBDOX-TACE in Taiwan. The conclusions of this expert panel are described in the following article.

(c) 2018 S. Karger AG, Basel

\section{Introduction}

Transarterial chemoembolization (TACE) that incorporates an oil-based emulsion agent is well established in the treatment of hepatocellular carcinoma (HCC) patients who are not suitable for surgery. The development of drug-eluting beads (DEBs) as a substitute for oil emulsion is a relatively novel approach which has shown great clinical promise. Six years have passed since the first widely accepted technical recommendations on HCC treatment with DEBs were published by Lencioni et al. [1], and new studies in the intervening years have contributed to a better understanding of when and how to apply this new therapeutic modality. Additionally, important differences in terms of underlying liver pathology and the practice of transcatheter embolization exist between Asian populations and the studies cited in Lencioni et al.'s [1] guideline. To address these concerns, we convened a panel of experts specializing in different aspects of HCC treatment to craft an updated set of recommendations that better reflects recent clinical experiences and is tailored to the use of DEBs in Taiwan.

HCC is the predominant primary liver cancer in many countries and is the third most common cause of cancer-related death in the Asia-Pacific region. In the Asia-Pacific region, chronic hepatitis $B$ virus (HBV) and hepatitis $C$ virus infections are the main etiological agents; in particular, chronic hepatitis B is still the major cause in all Asia-Pacific countries except Japan. Chronic hepatitis C is an important cause of HCC in Japan, representing 70\% of HCCs. Over the past several decades, the prevalence of chronic hepatitis $\mathrm{C}$ has been rising in many Asia-Pacific countries [2]. The highest age-adjusted incidence rates ( $>20$ per 100,000) of HCC are recorded in East and South-East Asia (North and South Korea, China, Hong Kong, Taiwan, Thailand, Vietnam, and Philippines) and in sub-Saharan Africa, which accounts for $82 \%$ of liver cancer cases worldwide. In particular, 55\% of all HCC cases worldwide are reported from China [3]. A medium-to-high incidence rate is found in Southern Europe, whereas lowincidence areas $(<5$ per 100,000) are found in South and Central America and the rest of Europe [4].

HCC is the second leading cause of cancer-related death in Taiwan, the majority of cases being associated with chronic HBV infection [5]. With an estimated $75 \%$ of the more than 350 million HBV carriers worldwide living in the Asia-Pacific region, including Taiwan [6, 7], HCC remains a serious global health-care issue. More than $70 \%$ of new liver cancer cases diag- 
Chang et al.: Multidisciplinary Taiwan Consensus Recommendations for the Use of DEBDOX-TACE in Hepatocellular Carcinoma Treatment

nosed annually were found in Asia [8], a region that also contains an estimated $75 \%$ of the global burden of chronic HBV patients [3]. Chronic HBV infection is the main cause of HCC in Asia, where the virus is endemic and vertical transmission is common. Japan, Saudi Arabia, Egypt, and Pakistan are some of the few exceptions due to the high prevalence of hepatitis $\mathrm{C}$ virus infection in these countries $[9,10]$.

While surgical resection continues to be a mainstay of treatment in appropriately selected patients, less invasive techniques, such as TACE, have become accepted worldwide as an effective treatment for patients with unresectable HCC [11,12]. Previous studies have shown that staging of HCC based on the tumor-node-metastasis (TNM) system and therapy selection via the Barcelona Clinic Liver Cancer (BCLC) staging system are also applicable in the Taiwanese population and convey survival benefits [13-15]. First introduced in 1980, TACE is based on the principle of delivering a high local concentration of chemotherapeutic drug via the hepatic arterial infusion, followed by embolization or reduction in arterial flow using various types of particles. The surrounding liver parenchyma is spared due to its preferential blood supply by the portal venous circulation. The reduction in arterial inflow is conceptually appealing because it directly causes some degree of ischemia within the tumor, which may induce tumor cell death, and significantly extends tumor drug concentrations. As a result, systemic toxicity is reduced since most of the injected chemotherapeutic agent will remain within the tumor bed rather than escaping to the systemic circulation. In the hands of an experienced operator, therapeutic effects can be achieved with minimal systemic toxicity [16].

In spite of its physiological advantages, preservation of liver function remains a major concern after conventional TACE (cTACE) with oil emulsion-based agents. An increase in liver enzymes after embolization is well documented and indicates variable levels of hepatocyte injury [17]. A potential complication of TACE is post-treatment HBV reactivation, and careful monitoring of viral load is needed. Recent studies have found that administration of nucleoside analogs can effectively inhibit HBV reactivation in post-TACE patients and can reduce the rate of liver function deterioration and liver failure. Toyoda et al. [18] have reported that the use of nucleoside analogues therapy could also lead to longer survival in patients with HBV-associated HCC who were treated with TACE. Another important limitation of cTACE is inconsistency in the technique and arrangement of embolization schedules, which is caused in part by the unpredictable duration for which chemotherapeutic agents remain within each tumor. To further improve patient tolerance of TACE and sustain high concentrations of chemotherapeutic agents within tumors, DEBs have been developed to complement conventional treatment regimens. Recent clinical studies have shown that DEBs loaded with doxorubicin have a safe pharmacokinetic profile with lower systemic drug exposure and significantly reduced liver toxicity compared to CTACE $[16,19,20]$. Importantly, doxorubicin levels remain detectable in the liver parenchyma for up to 1 month after embolization [12].

DC Bead (Biocompatibles UK Ltd.) is a relatively new embolic agent that consists of microspheres, which can be used on its own or in conjunction with doxorubicin. In a multicenter randomized controlled trial, investigators treating HCC patients with DEB doxorubicin (DEBDOX)-TACE found a significant decrease in liver toxicity and drug-related adverse events compared to cTACE [16]. There is also mounting evidence that DEBDOX-TACE treatment response rates for individuals with unresectable single tumors or multiple tumors (intermediate stage), Child-Pugh class B cirrhosis, or recurrent HCC are higher than can be consistently achieved with cTACE [16, 20-22]. In an Asian case-control study, Song et al. [23] reported that patients with larger $(>5 \mathrm{~cm})$ or multiple HCC tumors responded better to DEBDOX-TACE than to cTACE. Given its favorable clinical response and potential to reduce complications, TACE regimens utilizing DEBDOX-TACE are increasingly being considered as first-line treatment tools for HCC [24-27]. Even though treatment efficacy of DEBs in advanced-stage HCC (i.e., BCLC stage C) has not been extensively studied, preliminary experience suggests that patients 
with Child-Pugh class A disease with advanced disease may fare better with aggressive locoregional treatment in the form of TACE with DEBs than with systemic monotherapy with sorafenib. Furthermore, based on the results of some recent studies, we might conclude that TACE with DEBs could improve the clinical effectiveness in patients with more advanced HCC and be safe in high-risk patients [28-31].

Despite the clinical success of both cTACE and more recent DEBs, an important issue remains to be addressed in daily practice. Multiple variations of TACE protocols remain in use around the world, mainly revolving around the number and type of chemotherapeutic agents used, type of embolic material, reliance on Lipiodol, and selectivity of catheter positioning. As a result, it has been difficult to interpret and compare results obtained from different centers in a systematic fashion. This article summarizes the conclusions of a panel of experts convened to establish a possible consensus on standardized TACE regimens based on DEBDOX-TACE in Taiwan.

\section{Consensus Recommendations on the Use of DEBDOX-TACE in HCC Treatment}

A multidisciplinary panel of physicians with expertise in the fields of interventional radiology, gastroenterology, surgery, and oncology, all of whom have experience with the use of DEBDOX-TACE in HCC treatment, gathered to develop the following technical recommendations. This document presents a summary of the consensus recommendations of our multidisciplinary expert panel. All members have considerable experience in the field of interventional oncology and include interventional radiologists, general surgeons, gastroenterologists specializing in hepatology, as well as oncologists. Guidelines were generated based on the Oxford Center for Evidence-Based Medicine (OCEBM) (March 2009) (including level of evidence/recommendation) (Table 1; online suppl. Appendix 1; for all online supplementary material, see www.karger.com/doi/10.1159/000487608) [22] and a face-to-face meeting, while the manuscript was prepared by the lead author and approved by the entire group prior to submission. Although these recommendations may serve as a set of general guidelines, each patient undergoing TACE presents with a unique combination of individual and tumor characteristics that must be carefully assessed, and the best approach can only be decided on by the interventional radiologist treating the patient.

\section{Patient Selection (Level of Evidence: 1b/Recommendation: A)}

At present, DEBDOX-TACE use in TACE is primarily indicated in patients who are unable to receive curative treatment via surgery, liver transplantation, or percutaneous ablation, which is similar to cTACE. Based on consensus recommendations on HCC treatment by the Asian Pacific Association for the Study of the Liver, TACE is recommended as a first-line treatment for unresectable, large, or multifocal HCCs without vascular invasion or extrahepatic spread (1b/A). TACE can also be selectively performed in early-stage patients for whom radiofrequency ablation is unsuitable due to either tumor location or medical comorbidities (3/C) [32]. Besides the indications it shares with cTACE, DEBDOX-TACE can also be considered as a salvage embolizing agent when HCC has proven refractory to cTACE [33].

There is currently no consensus on maximum treatable tumor size for TACE with DEBDOX-TACE, and size should not be considered an absolute exclusion criteria. The most important factor to consider is postembolization liver function, which depends in large part on the amount of remaining liver volume that is preserved. Rather than considering the absolute size of the tumor in relation to the liver, an assessment should be made based on a patient's pretreatment liver function and the percentage of functional liver parenchyma that would remain after TACE. In general terms, a pretreatment Child-Pugh classification score of 


\section{Liver

Table 1. Summary guidance of Oxford Center for Evidence-Based Medicine (OCEBM) literature review ${ }^{\mathrm{a}}$ [22]

\begin{tabular}{|c|c|}
\hline Level & Evidence (LoE) guidance \\
\hline \multirow[t]{3}{*}{1} & 1a: SR or RCT \\
\hline & 1b: individual RCT \\
\hline & 1c: all or none \\
\hline \multirow[t]{3}{*}{2} & 2a: SR of cohort studies \\
\hline & 2b: individual cohort study \\
\hline & 2c: "outcomes" research \\
\hline \multirow[t]{2}{*}{3} & 3a: SR of case-control studies \\
\hline & 3b: individual case-control studies \\
\hline 4 & Noncomparative studies: case series, case report, or not well-designed clinical studies \\
\hline 5 & Opinion of respected authorities, descriptive epidemiology, or report of expert committee \\
\hline Level & Recommendation (R) guidance \\
\hline A & Strongly recommended \\
\hline B & Recommended \\
\hline $\mathrm{C}$ & Considerable but insufficient evidence \\
\hline $\mathrm{D}$ & Not recommended \\
\hline
\end{tabular}

A is recommended, but superselective embolization may still be considered even in Child Pugh class B [16].

If vascular invasion in the form of portal vein thrombosis is present, we recommend the following guidelines based on Watanabe et al.'s [34] grading scheme. There are 5 grades in total, Vp0 to Vp4, which correspond to progressively more central thrombosis of portal venous branches. Patients with Vp1 or Vp2 levels of portal thrombosis may still be treated with DEBDOX-TACE, while Vp3 cases should receive complementary radiotherapy. The presence of severe portal vein thrombosis $(\mathrm{Vp} 4)$ is a relative contraindication. In all cases with portal vein thrombosis, other therapeutic modalities, such as Yttrium-90, hepatic artery infusion chemotherapy, and target therapy, should be considered in addition to TACE.

\section{Pre-Treatment Imaging [24] (Level of Evidence: 1a/Recommendation: A)}

All patients should undergo either abdominal contrast-enhanced computed tomography (CT) with 3- or 4-phase image acquisition or magnetic resonance imaging (MRI). This allows the physician to evaluate whether there is indication for transcatheter treatment of HCC with DEBDOX-TACE and to establish an individualized treatment approach. To accurately assess the number, size, and location of hepatic lesions, both CT and MR scans should include unenhanced, arterial and portal venous phases. Utilizing appropriate contrast injection rates and optimal scanning protocols is crucial for separating individual imaging phases, which will allow for a high HCC detection rate and identification of benign lesions that may coexist within the liver. Any extrahepatic involvement either within the abdomen or in distant organs should also be sought via additional examinations.

Loading Dose of Doxorubicin [30] (Level of Evidence: 1a/Recommendation: A)

DC Bead microspheres are packaged in 2-mL vials of hydrated beads in sodium phosphate solution. Approximately 50-75 mg doxorubicin is loaded into each vial of DC Bead, 


\section{Liver Cancer}

resulting in a loading dose of 25-37.5 mg doxorubicin per $\mathrm{mL}$ of beads. The maximum dose for a single treatment session has been set at $150 \mathrm{mg}$, based on a study showing that this was the maximum acceptable dose for local infusion of doxorubicin. Up to a day prior to planned DEBDOX-TACE treatment, the hospital pharmacy will load the desired chemotherapy agent. This is achieved by first making a solution of the drug in isotonic saline to the desired concentration, then injecting it into the vial containing a slurry of DC Bead from which the packing solution has been removed. Depending upon the selected bead size range, drug uptake onto the DC Bead will occur between 30 min and $2 \mathrm{~h}$ (small beads load more quickly due to surface area effects). Since maximum drug loading capacity is controlled by the number of drug-binding sites in the beads, the maximum dose achievable per volume is dependent on the molecular mass of the chemotherapeutic drug. In general, we recommend a maximum load of $75 \mathrm{mg}$ of doxorubicin in a single 2-mL vial of DC Bead. The loading time (to reach $98 \%$ uptake) for $75 \mathrm{mg}$ of doxorubicin per vial of 100 - to $300-\mu \mathrm{m}$ DC Bead is 60 min, while 300 - to $500-\mu \mathrm{m}$ and 500 - to $700-\mu \mathrm{m}$ beads take 90 and $120 \mathrm{~min}$ to load, respectively.

\section{Dosage Planning for Doxorubicin (Level of Evidence: 2b/Recommendation: B)}

The doxorubicin dose infused during each DEBDOX-TACE should be adjusted on an individualized basis and tailored to a patient's current disease status. Although we cannot make an absolute dose recommendation that is appropriate for every patient, the interventional radiologist may be guided in part by comparing the patient's disease status with the Milan criteria proposed for liver transplantation (solitary tumor $\leq 5 \mathrm{~cm}$ or a maximum of 3 tumors each $\leq 3 \mathrm{~cm}$ ). For limited HCC within the Milan criteria, the treatment strategy may include escalation of doxorubicin dose up to $75 \mathrm{mg}$, loaded into each vial of DC Bead during a single TACE. For advanced disease, exceeding the Milan criteria, the doxorubicin dose may be increased to a maximum of $150 \mathrm{mg}$ loaded into 2 vials of DC Bead. If both lobes of the liver are involved and the tumors are large, each lobe can be embolized in separate treatment sessions 4 weeks apart. Delay in subsequent treatment may be required should serious complications arise. Liver enzymes should be allowed to return to baseline before performing the second treatment session. Tumors in both hepatic lobes may be embolized in a single session, with the dose being split according to the extent of tumor burden.

In case a solitary or unilobar HCC is bulky, it should be approached in a similar manner as bilobar tumors. Two sessions separated by a 4-week recovery period are recommended in most cases unless the tumor has replaced a majority of the liver parenchyma, in which case an additional management strategy should be contemplated. In addition, for extremely hypervascular tumors where flow stasis cannot be achieved by DEBDOX-TACE alone, additional embolizers with unloaded beads of appropriate size combined with Gelfoam may be applied. It should be noted that this process should only be attempted by experienced interventional radiologists, and great care must be taken to avoid excessive embolization resulting in permanent injury to local biliary and vascular systems.

Contrast material pooling in the target tumor bed is referred to as a vascular lake and is often seen during TACE with DEB microspheres for patients with HCC [16]. These vascular lakes are theorized to be blood-filled sinuses within the tumor resulting from extravasation and show possible correlation with tumor size and treatment response [35]. Although a consensus on the management of these cavities during TACE has yet to be established in the literature, all members of this panel unanimously recommended that Gelfoam be administered after DEBDOX-TACE injection, in order to achieve complete embolization in tumors with one or more vascular lakes [36]. 
Chang et al:: Multidisciplinary Taiwan Consensus Recommendations for the Use of DEBDOX-TACE in Hepatocellular Carcinoma Treatment

\section{Choice of DC Bead Size (Level of Evidence: 2b/Recommendation: B)}

DC Bead is commercially available in 4 sizes: 70-150, 100-300, 300-500, and 500-700 $\mu \mathrm{m}$. While the selection of DC Bead size is guided by tumor vascularity and the size of tumorfeeding arteries, use of 100 - to $300-\mu \mathrm{m}$ beads is recommended as a reference. Beads with smaller diameters can be delivered more readily into distal tumor-feeding vessels, while larger beads tend to become lodged in more proximal arteries where they may cause thrombosis [37]. If the larger feeding hepatic arteries become occluded and inaccessible, future TACE procedures may be rendered much more difficult. Therefore, we recommend using the smaller 100- to 300- $\mu \mathrm{m}$ beads, which allows good tumor penetration while preserving normal hepatic feeding arteries [38].

Although the best bead size range for each treatment will be unique to the patient, in general, if the tumor is smaller than $3 \mathrm{~cm}$ in diameter, DC Bead M1 $(70-150 \mu \mathrm{m})$ is recommended. If this is unavailable, then 100 - to $300-\mu$ m beads can be applied. For very large tumors, we suggest 2 vials of beads be used in combination, one being 100-300 $\mu \mathrm{m}$ and the other being 300-500 $\mu \mathrm{m}$ in size.

HCC with an arteriovenous shunt confers an increased risk of pulmonary complications during TACE. Special care should be taken to choose the appropriate bead size in these patients, and Gelfoam embolization to obliterate tumor feeders should be considered. Moreover, larger HCCs may be accompanied by intratumoral arterioportal or arteriovenous shunts, and caution should be taken when using the smaller sizes of embolic particles to avoid hepatic and pulmonary injury [39]. It is recommended that embolization with Gelfoam pledgets be conducted for patients with significant hepatic arterioportal or arteriovenous shunting prior to consideration for DEBDOX-TACE treatment. Even if occlusion of surrounding shunts has been confirmed by angiography, DEBDOX-TACE beads at least $100-300 \mu \mathrm{m}$ in size may still be a better choice in these patients.

Preparation of DC Bead for Injection [30] (Level of Evidence: 1a/Recommendation: A)

A nonionic contrast medium should be used to deliver the doxorubicin-loaded DC Bead. Each $\mathrm{mL}$ of DC Bead should be mixed with at least 5-10 mL of nonionic contrast (i.e., 1 vial of DC Bead into 10-20 mL of nonionic contrast) before injection.

\section{Catheter Positioning (Level of Evidence: 2b/Recommendation: B)}

Prior to each embolization, angiography of the hepatic and superior mesenteric artery should be performed to demonstrate liver arterial anatomy and feeding arteries to the tumor, as well as to check for obvious portal or venous shunts. C-arm cone-beam CT (CBCT) with rotational angiography may be used to identify tumor arterial supply with greater confidence, while the multiplanar reconstructions obtained during rotational imaging can help guide catheter tip positioning. For the segmental or subsegmental approach, a microcatheter should be superselectively positioned into the feeding vessels if at all possible, and all of the vessels feeding the tumor must be accurately located [40]. The application of microcatheters reduces the incidence of vasospasms and ensures forward flow of embolic materials during DC Bead delivery, although the operator should still take care not to wedge the catheter tip too tightly so as to avoid reflux along the catheter shaft.

Larger tumors may require a lobar approach, in which case a catheter is placed as selectively as possible into the left or right hepatic artery while avoiding the origin of arterial branches supplying extrahepatic organs, such as the cystic artery. To prevent inadvertent reflux of embolic particles into these branches, they must be bypassed either by extending the catheter tip well past the vessel origins or, if this is not feasible, by embolizing the arteries with coils or gelatin sponge prior to DEBDOX-TACE administration. Once injection begins, forward flow of beads into the target vessel must be continuously monitored to prevent complications. 


\section{Liver

Chang et al.: Multidisciplinary Taiwan Consensus Recommendations for the Use of DEBDOX-TACE in Hepatocellular Carcinoma Treatment

When used in conjunction with conventional selective and superselective angiography, this technique can also more accurately delineate the distribution of embolic materials. Therefore, we also recommend repeating CBCT or angio-CT immediately after DC Bead delivery, in order to confirm suitable tumor saturation and occlusion of feeding vessels [41].

\section{Injection Rate [30] (Level of Evidence: 1a/Recommendation: A)}

DEBDOX-TACE injection must be slow and steady, with the rate determined by flow within the target vessel. Periodic rotation of the bead-containing syringe helps prevent beads from sinking to the bottom, or a 3-way stop cock may be used to maintain suspension of DC Bead within its nonionic contrast medium.

\section{Embolization End Point (Level of Evidence: 2b/Recommendation: B)}

The end point of embolization is stasis (complete obliteration of tumor stains on digital subtraction angiography) or near-stasis (absence of contrast flow in the tumor-feeding artery within a span of 2-5 heart beats) [42] of flow in the segmental artery that directly feeds the target tumor, at which point further DEBDOX-TACE injection should cease. While the absence of tumor stain on postembolization angiography is the primary guide of flow stasis, the appropriate end point may be different during each treatment depending on patient and tumor factors. First of all, the catheter tip position during DEBDOX-TACE injection and follow-up angiography certainly influences the appearance of stasis, and the tip should be placed so as to show preserved arterial flow to normal liver parenchyma as well as absence of tumor stain. The flow rate of follow-up angiography should also be similar or slower than that used during diagnostic imaging. Another issue to consider is the patient's baseline cirrhosis status, which affects whether lobar, segmental, or subsegmental embolization can safely be considered. In any case, the operator's experience and clinical decision should be the final guide in determining the embolization end point.

Regardless of the amount of DEBDOX-TACE actually administered after the end point is reached, reflux of embolic particles due to excess infusion must be avoided. It has been previously reported that complete stasis of a target artery may lead to an increase in adverse effects [43-45], including increased liver toxicity [46] and expression of angiogenic growth factors that may induce HCC disease progression [47-49]. If the end point is not achieved after injection of the scheduled volume of loaded DC Beads, 1 of 3 separate choices may be taken. One option is to inject additional unloaded beads until near-stasis is attained. Another choice would be to infuse Gelfoam instead of beads until complete obliteration of tumor stain is observed on angiography. This recommendation is based on the treatment experience of members in our expert panel and differs from the previous guideline by Lencioni et al. [1]. The other choice is to refrain from injecting additional embolic material and instead schedule the patient for a repeat treatment after an interval during which imaging follow-up can be arranged.

\section{Post-Treatment Imaging (Level of Evidence: 1a/Recommendation: A)}

To evaluate tumor response and plan further therapy, liver dynamic CT or MRI should be performed 4-6 weeks after a course of DEBDOX-TACE. Use of the modified Response Evaluation Criteria in Solid Tumors (mRECIST) guideline for HCC response classification [50] is recommended to assess response and predict patient outcome. Individuals for whom complete response was not attained should be considered for repeat treatment with DEBDOX-TACE, except in cases where technical difficulties, such as small or tortuous feeding arteries, prohibit further TACE. Embolization may be scheduled after 4-8 weeks of recovery, in the absence of contraindications and with confirmation that liver enzymes have returned to baseline levels. For patients with no evidence of residual viable disease, imaging follow-up is recommended every 3 months. 
Chang et al.: Multidisciplinary Taiwan Consensus Recommendations for the Use of DEBDOX-TACE in Hepatocellular Carcinoma Treatment

Treatment Discontinuation (Level of Evidence: 1a/Recommendation: A)

If objective response in treated tumor(s) cannot be achieved after at least 2 courses of DEBDOX-TACE therapy, it is defined as untreatable progression, and bead embolization should cease. In this case, alternative management may be applied depending on tumor and patient condition. Although new intrahepatic tumor foci arising in a region distinct from previously treated tumors are clearly defined as tumor progression according to mRECIST, this does not contraindicate further treatment with DC Bead. Treatment should be discontinued if a patient experiences deterioration in clinical or functional status, more specifically if persistent hepatic decompensation occurs or if there is a progression in ECOG performance status $>2$ [1]. It should be noted that while this panel suggests a minimum number of DEBDOXTACE before determining whether it is an effective treatment, there is no maximum number of embolization as long as patient condition permits treatment and tumor control is achieved.

\section{Final Remarks}

The consensus recommendations given in this article represent the meticulous efforts of a panel of experts at instituting a consistent and effective standard for the treatment of HCC using DEBDOX-TACE. It is meant to serve as a general guideline rather than a strict set of rules, and the various tumor- and patient-related factors that affect each case should be considered in tailoring treatment to best suit individual needs. We firmly believe that interventional radiologists should have the final say in deciding on the technical aspects of each embolization procedure, while keeping the recommendations listed here in mind. In closing, we must stress that despite the generally improved patient tolerance of DEBDOX-TACE compared to CTACE, the operator must be cognizant at all times of the spectrum of potential adverse events and complications associated with this procedure so as to manage them appropriately.

\section{Acknowledgement}

The authors thank BTG International Asia Limited for its assistance in setting up the panel and Ivan Loh, BTG International Asia Limited, for providing editorial support for this article.

\section{Disclosure Statement}

The authors declare that they have no conflicts of interest.

\section{References}

1 Lencioni R, de Baere T, Burrel M, et al: Transcatheter treatment of hepatocellular carcinoma with doxorubicinloaded DC Bead (DEBDOX): technical recommendations. Cardiovasc Intervent Radiol 2012;35:980-985.

-2 Zhu RX, Seto WK, Lai CL, Yuen MF: Epidemiology of hepatocellular carcinoma in the Asia-Pacific region. Gut Liver 2016;10:332-339.

3 Lai CL, Ratziu V, Yuen MF, Poynard T: Viral hepatitis B. Lancet 2003;362:2089-2094.

$\checkmark 4$ Nordenstedt $\mathrm{H}$, White DL, El-Serag HB: The changing pattern of epidemiology in hepatocellular carcinoma. Dig Liver Dis 2010;42(suppl 3):S206-S214.

-5 Wu CY, Chen YJ, Ho HJ, et al: Association between nucleoside analogues and risk of hepatitis B virus-related hepatocellular carcinoma recurrence following liver resection. JAMA 2012;308:1906-1914.

-6 Changchien CS, Chen CL, Yen YH, et al: Analysis of 6,381 hepatocellular carcinoma patients in southern Taiwan: prognostic features, treatment outcome, and survival. J Gastroenterol 2008;43:159-170.

7 Liaw YF, Chu CM: Hepatitis B virus infection. Lancet 2009;373:582-592. 
8 Lunn RM, Zhang YJ, Wang LY, et al: p53 mutations, chronic hepatitis B virus infection, and aflatoxin exposure in hepatocellular carcinoma in Taiwan. Cancer Res 1997;57:3471-3477.

-9 Yuen MF, Hou JL, Chutaputti A; Asia Pacific Working Party on Prevention of Hepatocellular Carcinoma: Hepatocellular carcinoma in the Asia pacific region. J Gastroenterol Hepatol 2009;24:346-353.

10 But DY, Lai CL, Yuen MF: Natural history of hepatitis-related hepatocellular carcinoma. World J Gastroenterol 2008;14:1652-1656.

11 Llovet JM, Bruix J: Systematic review of randomized trials for unresectable hepatocellular carcinoma: chemoembolization improves survival. Hepatology 2003;37:429-442.

-12 Vogl TJ, Naguib NN, Nour-Eldin NE, et al: Review on transarterial chemoembolization in hepatocellular carcinoma: palliative, combined, neoadjuvant, bridging, and symptomatic indications. Eur J Radiol 2009;72: 505-516.

13 Kee KM, Wang JH, Wang CC, et al: Hepatocellular carcinoma associated with extra-hepatic primary malignancy: its secular change, clinical manifestations and survival. Sci Rep 2016;6:30156.

$\checkmark 14$ Kee KM, Wang JH, Lin CY, et al: Validation of the 7th edition TNM staging system for hepatocellular carcinoma: an analysis of 8,828 patients in a single medical center. Dig Dis Sci 2013;58:2721-2728.

15 Wang JH, Changchien CS, Hu TH, et al: The efficacy of treatment schedules according to Barcelona Clinic Liver Cancer staging for hepatocellular carcinoma - survival analysis of 3,892 patients. Eur J Cancer 2008;44:10001006.

-16 Lammer J, Malagari K, Vogl T, et al: Prospective randomized study of doxorubicin-eluting-bead embolization in the treatment of hepatocellular carcinoma: results of the PRECISION V study. Cardiovasc Intervent Radiol 2010;33:41-52.

17 Lewis AL, Gonzalez MV, Lloyd AW, et al: DC Bead: in vitro characterization of a drug-delivery device for transarterial chemoembolization. J Vasc Interv Radiol 2006;17(2 Pt 1):335-342.

18 Toyoda H, Kumada, T, Tada T, et al: Transarterial chemoembolization for hepatitis B virus-associated hepatocellular carcinoma: improved survival after concomitant treatment with nucleoside analogues. J Vasc Interv Radiol 2012;23:317-322.

-19 Varela M, Real MI, Burrel M, et al: Chemoembolization of hepatocellular carcinoma with drug eluting beads: efficacy and doxorubicin pharmacokinetics. J Hepatol 2007;46:474-481.

20 Vogl TJ, Lammer J, Lencioni R, et al: Liver, gastrointestinal, and cardiac toxicity in intermediate hepatocellular carcinoma treated with PRECISION TACE with drug-eluting beads: results from the PRECISION V randomized trial. AJR Am J Roentgenol 2011;197:W562-W570.

21 Malagari K, Pomoni M, Kelekis A, et al: Prospective randomized comparison of chemoembolization with doxorubicin-eluting beads and bland embolization with BeadBlock for hepatocellular carcinoma. Cardiovasc Intervent Radiol 2010;33:541-551.

22 OCEBM Levels of Evidence Working Group: The Oxford Levels of Evidence 2. Oxford, Oxford Centre for Evidence-Based Medicine, 2017. http://www.cebm.net/index.aspx?o=5653 (accessed November 18, 2017).

23 Song MJ, Chun HJ, Song DS, et al: Comparative study between doxorubicin-eluting beads and conventional transarterial chemoembolization for treatment of hepatocellular carcinoma. J Hepatol 2012;57:1244-1250.

24 Elsayes KM, Hooker JC, Agrons MM, et al: 2017 version of LI-RADS for CT and MR imaging: an update. Radiographics 2017;37:1994-2017.

-25 Martin R, Irurzun J, Munchart J, et al: Optimal technique and response of doxorubicin beads in hepatocellular cancer: bead size and dose. Korean J Hepatol 2011;17:51-60.

26 Namur J, Citron SJ, Sellers MT, et al: Embolization of hepatocellular carcinoma with drug-eluting beads: doxorubicin tissue concentration and distribution in patient liver explants. J Hepatol 2011;55:1332-1338.

$\checkmark 27$ Song MJ, Park CH, Kim JD, et al: Drug-eluting bead loaded with doxorubicin versus conventional Lipiodolbased transarterial chemoembolization in the treatment of hepatocellular carcinoma: a case-control study of Asian patients. Eur J Gastroenterol Hepatol 2011;23:521-527.

28 Kalva SP, Pectasides M, Liu R, et al: Safety and effectiveness of chemoembolization with drug-eluting beads for advanced-stage hepatocellular carcinoma. Cardiovasc Intervent Radiol 2014;37:381-387.

29 Prajapati HJ, Dhanasekaran R, El-Rayes BF, et al: Safety and efficacy of doxorubicin drug-eluting bead transarterial chemoembolization in patients with advanced hepatocellular carcinoma. J Vasc Interv Radiol 2013; 24:307-315.

30 Biocompatibles UK Ltd, a BTG International Group Company: Instruction for Use DC Bead ${ }^{\mathrm{TM}}$. Farnham, DC Bead $^{\mathrm{TM}}$ Instruction for Use CN00103.5 and CN000164.2, 2013.

31 Cheng AL, Kang YK, Chen Z, et al: Efficacy and safety of sorafenib in patients in the Asia-Pacific region with advanced hepatocellular carcinoma: a phase III randomised, double-blind, placebo-controlled trial. Lancet Oncol 2009;10:25-34.

-32 Omata M, Lesmana LA, Tateishi R, et al: Asian Pacific Association for the Study of the Liver consensus recommendations on hepatocellular carcinoma. Hepatol Int 2010;4:439-474.

-33 Liapi E, Geschwind JF: Transcatheter arterial chemoembolization for liver cancer: is it time to distinguish conventional from drug-eluting chemoembolization? Cardiovasc Intervent Radiol 2011;34:37-49.

-34 Watanabe T, Itabashi M, Shimada Y, et al: Japanese Society for Cancer of the Colon and Rectum (JSCCR) guidelines 2010 for the treatment of colorectal cancer. Int J Clin Oncol 2012;17:1-29.

35 Seki A, Hori S, Shimono C: Management of vascular lake phenomenon on angiography during chemoembolization with superabsorbent polymer microspheres. Jpn J Radiol 2015;33:741-748. 
Chang et al.: Multidisciplinary Taiwan Consensus Recommendations for the Use of DEBDOX-TACE in Hepatocellular Carcinoma Treatment

36 Seki A, Hori S, Kobayashi K, Narumiya S: Transcatheter arterial chemoembolization with epirubicin-loaded superabsorbent polymer microspheres for 135 hepatocellular carcinoma patients: single-center experience. Cardiovasc Intervent Radiol 2011;34:557-565.

-37 Malagari K: Drug-eluting particles in the treatment of HCC: chemoembolization with doxorubicin-loaded DC Bead. Expert Rev Anticancer Ther 2008;8:1643-1650.

38 Kloeckner R, Weinmann A, Prinz F, et al: Conventional transarterial chemoembolization versus drug-eluting bead transarterial chemoembolization for the treatment of hepatocellular carcinoma. BMC Cancer 2015;15: 465.

-39 Lee KH, Liapi E, Vossen JA, et al: Distribution of iron oxide-containing Embosphere particles after transcatheter arterial embolization in an animal model of liver cancer: evaluation with MR imaging and implication for therapy. J Vasc Interv Radiol 2008;19:1490-1496.

-40 Basile A, Carrafiello G, Ierardi AM, et al: Quality-improvement guidelines for hepatic transarterial chemoembolization. Cardiovasc Intervent Radiol 2012;35:765-774.

41 Virmani S, Ryu RK, Sato KT, et al: Effect of C-arm angiographic CT on transcatheter arterial chemoembolization of liver tumors. J Vasc Interv Radiol 2007;18:1305-1309.

42 Lencioni R, Petruzzi P, Crocetti L: Chemoembolization of hepatocellular carcinoma. Semin Intervent Radiol 2013;30:3-11.

43 Guiu B, Deschamps F, Aho S, et al: Liver/biliary injuries following chemoembolisation of endocrine tumours and hepatocellular carcinoma: Lipiodol versus drug-eluting beads. J Hepatol 2012;56:609-617.

44 Malagari K, Pomoni M, Spyridopoulos TN, et al: Safety profile of sequential transcatheter chemoembolization with DC Bead: results of 237 hepatocellular carcinoma (HCC) patients. Cardiovasc Intervent Radiol 2011;34: 774-785.

45 Jin B, Wang D, Lewandowski RJ, et al: Chemoembolization endpoints: effect on survival among patients with hepatocellular carcinoma. AJR Am J Roentgenol 2011;196:919-928.

46 Geschwind JF, Ramsey DE, Cleffken B, et al: Transcatheter arterial chemoembolization of liver tumors: effects of embolization protocol on injectable volume of chemotherapy and subsequent arterial patency. Cardiovasc Intervent Radiol 2003;26:111-117.

47 Kobayashi N, Ishii M, Ueno Y, et al: Co-expression of Bcl-2 protein and vascular endothelial growth factor in hepatocellular carcinomas treated by chemoembolization. Liver 1999;19:25-31.

-48 Xiong ZP, Yang SR, Liang ZY, et al: Association between vascular endothelial growth factor and metastasis after transcatheter arterial chemoembolization in patients with hepatocellular carcinoma. Hepatobiliary Pancreat Dis Int 2004;3:386-390.

49 Rhee TK, Young JY, Larson AC, et al: Effect of transcatheter arterial embolization on levels of hypoxia-inducible factor-1 $\alpha$ in rabbit VX2 liver tumors. J Vasc Interv Radiol 2007;18:639-645.

-50 Lencioni R, Llovet JM: Modified RECIST (mRECIST) assessment for hepatocellular carcinoma. Semin Liver Dis 2010;30:52-60. 